\title{
Hyperfine structure measurements in the IF B-X system
}

\author{
G. Gouédard $\left({ }^{1}\right)$, N. Billy $\left({ }^{1}\right)$, B. Girard $\left({ }^{2}\right)$ and J. Vigué $\left({ }^{2}\right)$ \\ (1) Laboratoire de Spectroscopie Hertzienne $\left({ }^{*}\right)$, Département de Physique de l'E.N.S., 24 rue \\ Lhomond, 75231 Paris Cedex 05, France \\ (2) Equipe de Dynamique des Edifices Atomiques (**), IRSAMC, Université Paul Sabatier, 118 \\ route de Narbonne, 31062 Toulouse Cedex, France
}

(Received 25 November 1991, accepted 18 December 1991)

\begin{abstract}
We have measured and analyzed the hyperfine structure of a large number of optical transitions belonging to the IF B-X system. These transitions sample a large range of rovibrational levels $\left(9 \leqslant v^{\prime \prime} \leqslant 18\right.$ in the $\mathrm{X}$ state, $0 \leqslant v^{\prime} \leqslant 10$ in the $\mathrm{B}$ state, with $\left.J \leqslant 125\right)$. The hyperfine constants deduced from the observed spectra present some interesting variations with the rovibrational levels. We analyze carefully the electric quadrupole coupling constant in the $\mathrm{X}$ state and show that its variation is a sensitive probe of the ionic character of the IF molecular bond.
\end{abstract}

\section{Introduction.}

We have measured the hyperfine structure of a number $(\approx 200)$ of lines belonging to the IF B-X band system, by a technique of Doppler-free saturation spectroscopy. Our first motivation was a need for precise hyperfine structure (HFS) data for lines observed in our study of the Laser induced fluorescence (LIF) excitation spectra of IF produced by the reaction :

$$
\mathrm{I}_{2}+\mathrm{F} \rightarrow \mathrm{IF}(\mathrm{X}, v, J)+\mathrm{I} .
$$

We have achieved a precise study of the state resolved differential cross-sections for this reaction, by an analysis of the Doppler profile of the excitation lines [1]. The shape of these lines is due both to the Doppler effect and to their HFS structure; we thus had to know precisely the latter in order to extract the former contribution.

Previous measurements of the IF hyperfine structure have concerned either the $v^{\prime \prime}=0,1,2$ vibrational levels of the $\mathrm{X}^{1} \Sigma^{+}$state by millimeter wave spectroscopy $[2,3]$, or the $v^{\prime}=3,4$ levels of the $\mathrm{B}^{3} \Pi\left(0^{+}\right)$state by microwave-optical double resonance [4]. Both types of experiments have given very precise values for the IF hyperfine constants in the $B$ and $X$

(*) Unité de recherche de l'Ecole Normale Supérieure et de l'Université Paris 6, associée au CNRS (U.R.A. 18).

(**) Supported by CNRS (SDI 6296), MEN-DRED Université Paul Sabatier and Région MidiPyrénées. 
states (electric nuclear quadrupole coupling constant $e q Q$ are magnetic spin-rotation coupling constant $C_{\mathrm{I}}$ for the Iodine nucleus), but for a very limited number of rovibrational levels. However they showed the rapid variations of these parameters.

Our own measurements are less precise but cover a much wider field of quantum numbers : $9 \leqslant v^{\prime \prime} \leqslant 18,0 \leqslant v^{\prime} \leqslant 10, J \leqslant 125$. As is well known, the measurements on high $(\geqslant 10) J$ values lines are limited by the $\Delta F=\Delta J$ selection rule; they give only the differences $\Delta e q Q\left(v^{\prime}, J^{\prime}, v^{\prime \prime}, J^{\prime \prime}\right)$ and $\Delta C_{\mathrm{I}}\left(v^{\prime}, J^{\prime}, v^{\prime \prime}, J^{\prime \prime}\right)$ between the hyperfine parameters of the B and X states. Fortunately, we have been able to study the HFS structure of low $J$ (in particular P(1) and $\mathrm{R}(0)$ ) lines. We have thus obtained partially decorrelated values of the $e q Q$ and $C_{\mathrm{I}}$ coefficients.

The study of the rovibrational dependence of the hyperfine coupling constants can be used to determine the variations of these quantities with the molecular internuclear distance. This variation is interesting as it contains important information. For instance it is well known [5] that the value of the quadrupole coupling constant $e q Q$ is very sensitive to the ionic character of the molecular bond ; this has, for example, been studied in detail through the family of the halogen and interhalogen molecules [6]. A natural extension of this idea is to use the variation of this constant with the internuclear distance to trace the corresponding variation of the ionic character of the bond, and simple arguments [7] can be used to predict this variation. Strong vibrational variations of the quadrupole coupling constant have also been observed in the $B$ state of other interhalogen molecules : this is, for example, the case in $\mathrm{ICl}$ [8]. The spinrotation constant carries another type of information: this quantity should vanish in a pure Hund's case a) $\Omega=0$ state. Therefore its value is due to various perturbations (local or global) by $\Omega=1$ states. Both types of perturbations appear in the present study.

\section{Experimental.}

As explained in the introduction, one of our goals was to measure the hyperfine structure of lines previously observed in our study of differential cross sections resolved according to the $(v, J)$ values in the reactive collision :

$$
\mathrm{I}_{2}+\mathrm{F} \rightarrow \mathrm{IF}(\mathrm{X}, v, J)+\mathrm{I} .
$$

This reaction is now well characterized [9], and in particular is known to produce IF molecules in the ground $X$ state with a very large rovibrational energy : in the conditions of our experiment levels up to $v=20$ were populated, with also a rotational population extending up to the exoenergicity limit. We therefore had to design a system based on the same chemical reaction and to maintain a low background pressure in order to avoid rotational relaxation. Our experiment used two uncollimated molecular beams facing each other at a distance of $20 \mathrm{~mm}$, and the laser beam passes at mid distance, where the IF density is expected to be the highest. The iodine molecular beam is produced by expansion of pure iodine vapor (at a pressure of $4 \mathrm{mbar}$ ) through a $0.2 \mathrm{~mm}$ diameter nozzle heated to $120^{\circ} \mathrm{C}$. The fluorine atoms were produced by a microwave discharge through a $\mathrm{F}_{2} / \mathrm{He}(10: 90)$ mixture, in an alumina tube, using an Evenson-type cavity [10]. This tube (8 $\mathrm{mm}$ i.d.) was connected to the reaction vessel by a $20 \mathrm{~cm}$ long Teflon pipe of $4 \mathrm{~mm}$ i.d.

We estimated that the two beams introduced comparable numbers of fluorine atoms and iodine molecules per unit time in the reaction zone $\left(3 \times 10^{18} \mathrm{~s}^{-1}\right)$. The reaction vessel was pumped by a $2400 \mathrm{l} / \mathrm{s}$ oil diffusion pump; a $3 \mathrm{~cm}$ diameter aperture reduced the pumping speed to about $100 \mathrm{l} / \mathrm{s}$, so that the total pressure was maintained at about $10^{-2} \mathrm{mbar}$. The IF density was thus estimated to be at most $10^{-3}$ mbar. As the IF nascent population is spread over 3000 rovibrational levels, the typical IF density in one of these levels is of the order of 
$10^{10} \mathrm{~cm}^{-3}$. Our design criterion was to have a mean free path of the order of $1 \mathrm{~cm}$, so that reaction occurs close to the nozzles, but without too much rotational relaxation. Attempts to use higher flow rates of iodine or fluorine produced an intense chemiluminescence which degraded the signal to noise ratio of the Doppler-free signals.

To observe these highly resolved spectra of the product IF molecules, we decided to use one of the various techniques of saturation spectroscopy : saturated absorption was not a good choice, considering the very low density of absorbers. We thus used the technique of the Intermodulated Fluorescence [11] which allowed the observation of the saturation spectra over a nearly zero Doppler-broadened background. The reaction zone was crossed by two counterpropagating laser beams intensity-modulated at frequencies $f_{1}$ and $f_{2}(120 \mathrm{~Hz}$, $1100 \mathrm{~Hz}$ ) by mechanical choppers. We observed the component of the fluorescence signal modulated at both frequencies, by a double frequency lock-in detection technique. Excitation of the IF molecules was performed using a monomode ring dye-laser $(700 \leqslant \lambda \leqslant 770 \mathrm{~nm}$ and $820 \leqslant \lambda \leqslant 860 \mathrm{~nm}$ ) ; the laser wavelength was continuously measured by a home-made lambdameter, and further carefully linearized by interpolation between the peaks of a stable Fabry-Perot interferometer of $75 \mathrm{MHz}$ free spectral range ; this Fabry-Perot is made of Invar, thermally compensated with copper and is maintained under vacuum; its temperature is stabilized to within $0.02{ }^{\circ} \mathrm{C}$. The fluorescence signals were observed with a photomultiplier following a short wavelength-pass filter and a large acceptance lens system (Melles Griot aspheric condenser $f / 0.7$ ). This anti-Stokes detection scheme has been used to minimize observation of fluorescence from the «cold $» I_{2}$ molecules present in the reaction chamber. The good knowledge of the IF excitation spectrum [12] in this spectral region allowed a direct identification of the observed lines.

For most of these lines, the distance between the extreme HFS components was about $700 \mathrm{MHz}$, and these components were generally isolated with individual widths of the order of $5 \mathrm{MHz}$ or less.

\section{Hyperfine structure of the lines.}

The hyperfine structure of the IF $B$ and $X$ states is due to the Iodine nuclear spin $\left(I_{1}=5 / 2\right)$ and to the Fluorine nuclear spin $\left(I_{2}=1 / 2\right)$. The general form of the hyperfine Hamiltonian is :

$$
H=H_{\mathrm{EQ}}(1)+H_{\mathrm{MD}}(1)+H_{\mathrm{MD}}(2)
$$

where $H_{\mathrm{EQ}}(1)$ is the interaction of the electric quadrupole moment of the iodine nucleus with the electrons and $H_{\mathrm{MD}}(i)$ describes the effective spin-rotation interaction of nucleus $i$. Because $I_{2}=1 / 2$, the fluorine nucleus has no electric quadrupole moment and its only interaction is a magnetic dipolar one. Before this work, no effect of the fluorine nuclear spin has been observed in the hyperfine structures of IF ; we have observed small effects which will be discussed later. Neglecting them in a first step, each rotational level is split in a series of $F$ sublevels $\left(\left|J-I_{1}\right| \leqslant F \leqslant J+I_{1}\right)$; there are $2 I_{1}+1=6$ sublevels as soon as $J \geqslant I_{1}$. The diagonal matrix elements of $H_{\mathrm{EQ}}(1)$ are :

$$
\left\langle J I_{1} F\left|H_{\mathrm{EQ}}\right| J I_{1} F\right\rangle=-e q Q \times \frac{\left[3 / 4 K(K+1)-I_{1}\left(I_{1}+1\right) J(J+1)\right]}{2 I_{1}\left(2 I_{\mathrm{I}}-1\right)(2 J+3)(2 J-1)}
$$

where

$$
K=F(F+1)-I_{1}\left(I_{1}+1\right)-J(J+1) \text {. }
$$

Then, because of the very high value of the Iodine quadrupolar energy, the coupling to $J \pm 2$ is not negligible, at the present level of accuracy. For low $J$ values $(J \leqslant 10)$ we take this 
into account by diagonalizing the rotational + hyperfine Hamiltonian in the $(J, J \pm 2)$ basis set ; for higher $J$ values the $J-J \pm 2$ interaction is introduced by second order perturbation. The magnetic dipolar nuclear interaction is vanishing in a pure case a) $\Omega=0$ state. Perturbation of this $\Omega=0$ state by $\Omega=1$ states induces an effective term which can be written :

$$
\left\langle J I_{1} F\left|H_{\mathrm{MD}}\right| J I_{1} F\right\rangle=C_{\mathrm{I}} \mathrm{I}_{1} \cdot \mathbf{J}=\left(C_{\mathrm{I}} / 2\right)\left[F(F+1)-J(J+1)-I_{1}\left(I_{1}+1\right)\right] .
$$

For high $J$ levels the diagonal hyperfine interactions may be written in the simplified form :

$$
\begin{aligned}
& \left\langle J I_{1} F\left|H_{\mathrm{EQ}}(1)+H_{\mathrm{MD}}(1)\right| J I_{1} F\right\rangle= \\
& \quad \frac{-e q Q}{8 I(2 I-1)}\left[3 M^{2}+(3 M / J)\left(M(M+1)-I_{1}\left(I_{1}+1\right)+1 / 2\right)-I_{1}\left(I_{1}+1\right)\right]+C_{\mathrm{I}} M J
\end{aligned}
$$

with $M=(F-J)$.

The hyperfine structure of an optical transition between the B and X states of IF is thus the set of lines connecting the various $\left(F^{\prime}, F^{\prime \prime}\right)$ hyperfine levels for given $\left(J^{\prime}, J^{\prime \prime}\right)$ values. As the optical field does not act on the nuclear spins, the intensities of the different components are, in the linear regime, proportional to the square of the $(6 J)$ coefficients [13] :

$$
\left\{\begin{array}{lll}
J & F & I \\
J^{\prime} & F^{\prime} & 1
\end{array}\right\}
$$

For $J \gg I$ it is well known that the hyperfine transitions fall into three classes of rapidly decreasing intensities :

\begin{tabular}{|c|c|c|}
\hline Type & $\Delta F$ value & Intensity \\
\hline I & $\Delta F=\Delta J$ & 1 \\
\hline II & $\Delta F=0$ & $1 / J^{2}$ \\
\hline III & $\Delta F=-\Delta J$ & $1 / J^{4}$ \\
\hline
\end{tabular}

This is the origin of the so-called approximate $\Delta F=\Delta J$ selection rule. In very good experimental conditions, the $\Delta F=0$ and even the $\Delta F=-\Delta J$ lines could also be seen, but with much weaker intensities. We also want to recall that these intensity rules are valid only for high $J$ values $\left(J, J^{\prime} \gg I\right)$; for low $J$ all the allowed transitions may have intensities of the same order of magnitude.

Returning to the case of the high $J$ values, the spectra consist only of the six main $\Delta F=\Delta J$ hyperfine lines; then we only have access to the differences in the $e q Q$ and $C_{\mathrm{I}}$ values in the $\mathrm{X}$ and $\mathrm{B}$ states : $\Delta e q Q=e q Q^{\prime}-e q Q^{\prime \prime}$ and $\Delta C_{\mathrm{I}}=C_{\mathrm{I}}^{\prime}-C_{\mathrm{I}}^{\prime \prime}$, but not to the individual coefficients. This is a particular nuisance as we observed in the $B$ and $X$ states ranges of $(v, J)$ levels disconnected from the narrow ranges previously studied [2-4]. An example of such a « high $J »$ spectrum is given in figure 1. Fortunately, we have also observed very low $J$ lines in the spectrum. Of particular importance was the observation of several $R(0)$ and $\mathrm{P}(1)$ lines; they connect $J=0$ and $J=1$ rotational levels where obviously only the $J=1$ level has a hyperfine structure splitting the $F=3 / 2,5 / 2,7 / 2$ levels. These structures give therefore a direct measurement of the $e q Q$ values in the $\mathrm{X}$ (for $\mathrm{P}(1)$ ) or the $\mathrm{B}$ (for $\mathrm{R}(0)$ ) 
$\Delta \mathrm{F}=\Delta \mathbf{J}$ Lines

$\mathbf{F}-\mathbf{J}=$

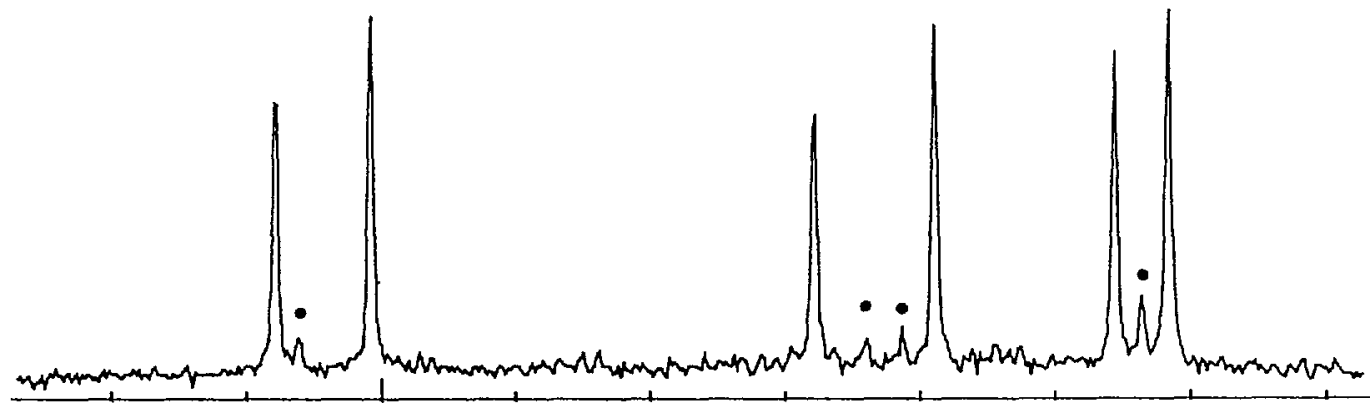

$100 \mathrm{MH} \iota$

Fig. 1. - Example of the Doppler-free spectrum of a « moderately high $J$ »IF B-X transition : the 6-13 $\mathrm{R}(25)$ transition. The spectrum is made of the six main $\Delta F=\Delta J$ lines which are labelled by their $(F-J)$ values, and also of some crossovers which are indicated by dots. The frequency scale unit is $100 \mathrm{MHz}$.

vibrational states; on the contrary the $C_{1} \mathbf{I}_{1} \cdot \mathbf{J}$ contribution is then too small to be measured, with our experimental accuracy.

We also observed for these low $J$ lines and up to $J \approx 10$, not only the expected main lines, but also crossover resonances [14], which appeared with intensities greater than the previously mentioned $\Delta F=0$ and $\Delta F=-\Delta J$ lines. Let us recall the main properties of these crossover resonances : they appear in a laser saturation experiment when two lines of frequencies $\nu_{1}$ and $\nu_{2}$ share a common (upper or lower) level; the crossover has a frequency $\nu_{\mathrm{c}}=\left(\nu_{1}+\nu_{2}\right) / 2$ (neglecting light shifts induced in the very high saturation regime as well as recoil effects). More importantly yet the crossover has an intensity roughly proportional to the geometrical mean of the parent lines : $I_{\mathrm{c}} \approx \sqrt{I_{1} I_{2}}$. In our context, crossover lines cannot exist between the main $\Delta F=\Delta J$ lines which do not share any common hyperfine level. But it is clear that crossovers may exist between the main $(\Delta F=\Delta J)$ lines and the $\Delta F=0$ and $\Delta F=-\Delta J$ lines. As to the intensities, it is readily seen that the crossovers between type I and type II lines have intensities proportional to $(1 / J)$. They are therefore more easily seen than the direct type II or III lines. On the whole, for $J, J^{\prime}>I=5 / 2$ there are 15 direct HFS lines, and 26 crossover lines, i.e. a total of 41 hyperfine lines, some of them being very weak or overlapped. The structure having a width of less than $1 \mathrm{GHz}$, the detection and assignment of all those lines would be very difficult. In practice, we have observed that for $J \geqslant 8$ the 6 type I lines are prominent, and some crossovers easily observable. For $8 \geqslant J>1$ the spectra contained a wealth of lines of comparable intensities, a number of them being blended. The situation evidently simplifies very much for the $\mathrm{R}(0)$ and $\mathrm{P}(1)$ lines where there is only 3 direct $\left(F=5 / 2\right.$ to $F^{\prime}=3 / 2,5 / 2,7 / 2$ or vice versa) lines and 3 crossovers. An example of such a spectrum is given in figure 2 .

If we include the effect of the Fluorine nuclear spin $I_{2}=1 / 2$ the levels can be described by the following coupling scheme:

$$
\left|\left(I_{1}\right) F I_{2} G\right\rangle
$$




$\begin{array}{rllrll}F^{\prime}=5 / 2 & F^{\prime \prime}= & 3 / 2 & & 7 / 2 & 5 / 2 \\ & \text { line } & 1 & 2 & & 3 \\ & \text { crossovers } & & 1-2 & & 2-3\end{array}$

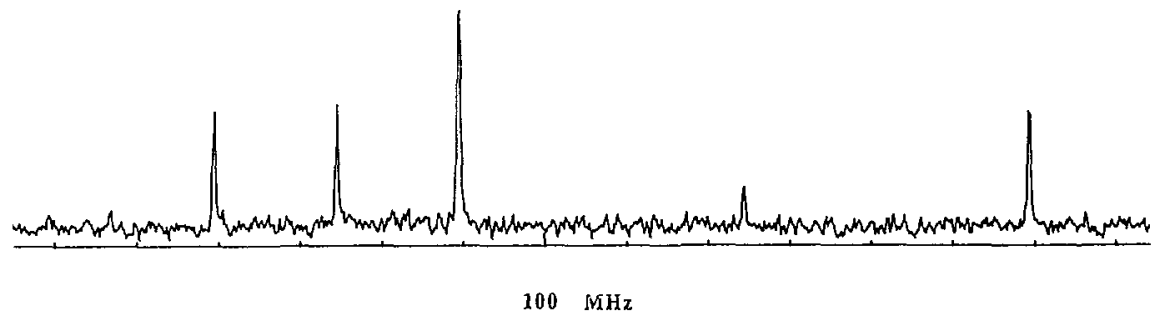

Fig. 2. - Hyperfine structure of a low- $J$ transition : the 7-14 $P(1)$ line. The three direct lines are labelled by their $F^{\prime \prime}$ values, and numbered. Only two crossovers are apparent on the figure and are labelled by the numbers of the parent direct lines.

where $\mathbf{G}$ is the true total angular momentum. Each level $F$ is then split in two components $G=F \pm 1 / 2$ by the fluorine magnetic dipolar interaction :

$$
\left\langle J I_{1} F I_{2} G\left|H_{\mathrm{MD}}(2)\right| J I_{1} F I_{2} G\right\rangle=C_{\mathrm{F}} \mathbf{I}_{2} \cdot \mathbf{J} \approx C_{\mathrm{F}} J(G-F)
$$

where the last equality is a good approximation at high $J$ values. We have indeed observed that lines at high $J$ values show some evidence of a splitting, just at the limit of our resolution. For example, we have observed a clear splitting of the components of the 5-16 P(84) line; we can deduce an approximate value of $\Delta C_{\mathrm{F}}=C_{\mathrm{F}^{\prime}}-C_{\mathrm{F}^{\prime \prime}} \approx 30 \mathrm{kHz}$ for this transition. This will be discussed below.

\section{Experimental results.}

We thus measured the HFS structure of $\approx 200$ lines in the IF (B-X) system. The bands inside which lines were measured are indicated in table I. These measurements therefore cover the

Table I. - Data field; the bands inside which we have measured Doppler-free hyperfine structures are marked by an asterisk $\left(^{*}\right)$.

\begin{tabular}{|c|c|c|c|c|c|c|c|c|c|c|c|}
\hline$V^{\prime \prime} / V^{\prime}$ & 0 & 1 & 2 & 3 & 4 & 5 & 6 & 7 & 8 & 9 & 10 \\
\hline $\begin{array}{r}9 \\
10 \\
11 \\
12 \\
13 \\
14 \\
15 \\
16 \\
17 \\
18 \\
19\end{array}$ & $*$ & $\begin{array}{l}* \\
*\end{array}$ & $*$ & $*$ & $\begin{array}{l}* \\
* \\
*\end{array}$ & $*$ & $\begin{array}{l}* \\
*\end{array}$ & $\begin{array}{l}* \\
*\end{array}$ & $\begin{array}{l}* \\
*\end{array}$ & $*$ & $*$ \\
\hline
\end{tabular}


ensemble of unpredissociated levels (and also the beginning of some predissociated regions) in the B state of IF $\left(v^{\prime} \leqslant 10\right)$; on the contrary, we have only observed levels belonging to $9 \leqslant v^{\prime \prime} \leqslant 19$ in the $\mathrm{X}$ state.

For a given rotational transition we substracted the mean of the observed HFS components and fitted the remaining independent line intervals : for example for high $J$ lines there were 6 HFS lines and so 5 independent intervals. Then the analysis differed according to the $J$ value of the transition under study:

- for high $(\geqslant 10) J$ lines, only $\Delta e q Q$ and $\Delta C_{\mathrm{I}}$ could be determined,

- for $\mathrm{R}(0)$ or $\mathrm{P}(1)$ lines, we fitted directly the corresponding $e q Q$.

The line by line fitting of the HFS constants showed a mean square deviation of the adjustments of about 1.2 MHz. This is the result of the laser frequency jitter, and of the irregularities in the laser frequency scans.

Examination of these results immediately showed a very important variation of the $e q Q$ and $C_{\mathrm{I}}$ parameters as functions of the $(v, J)$ quantum numbers in the $\mathrm{X}$ and, mainly, the $B$ states. We first tried to fit these variations with polynomials in powers of $(v+1 / 2)$ and $J(J+1)$; we were unable to fit the results corresponding to $v_{\mathrm{B}} \geqslant 8$ in the $\mathrm{B}$ state, as they show too rapid a variation. We thus limited the fits to $v_{\mathrm{B}} \leqslant 7$. The resulting parameters appear in table II ; the mean square deviation given by this model is $1.6 \mathrm{MHz}$. This is slightly larger than the $1.2 \mathrm{MHz}$ obtained in the line by line fitting, because of the previously mentioned rapid variations of the parameters. To take into account these variations in the $B$ state we have been obliged to use partial high powers polynomial descriptions. One has also to remember that there is a complete correlation between the purely rotational (i.e. $\alpha J(J+1))$ contributions to the $e q Q$ and $C_{\mathrm{I}}$ parameters of the $\mathrm{B}$ and $\mathrm{X}$ states, as we only measure for high $J$ values $\Delta e q Q$ and $\Delta C_{\mathrm{I}}$ of $\left(v_{\mathrm{B}}, v_{\mathrm{X}}, J_{\mathrm{X}}\right.$, and $\left.J_{\mathrm{B}}=J_{\mathrm{X}} \pm 1 \approx J_{\mathrm{X}}\right)$.

Table II. - Least-square fit parameters describing the IF B-X hyperfine structures measured in this work. The quoted errors are one standard deviation. The validity limits for these parameters are: $0 \leqslant v^{\prime} \leqslant 7$ in the $B$ state, $9 \leqslant v^{\prime \prime} \leqslant 18$ in the $X$ state $0 \leqslant J \leqslant 125$ in both states.

\begin{tabular}{|c|c|c|c|}
\hline$e q Q(B)=$ & $e q Q(X)=$ & $C_{1}(B)=$ & $C_{\mathrm{I}}(X)=$ \\
\hline$\left|\begin{array}{lll}-540 .(4) & & \\
-5 .(1) & \left(v^{\prime}+1 / 2\right) & \\
-0.09(1) & \left(v^{\prime}+1 / 2\right)^{3} & \\
+1.8(4) \times 10^{-3} & J(J+1) \\
-8.2(8) \times 10^{-4}\left(v^{\prime}+1 / 2\right) & J(J+1)\end{array}\right|$ & $\begin{array}{ll}-3441 . & \text { (fixed) } \\
+4.5(4) & \left(v^{\prime \prime}+1 / 2\right) \\
+0.29(2) & \left(v^{\prime \prime}+1 / 2\right)^{2}\end{array}$ & $\begin{array}{l}0.206(5) \\
+9 .(1) \times 10^{-3}\left(v^{\prime}+1 / 2\right) \\
+1.6(1) \times 10^{-4}\left(v^{\prime}+1 / 2\right)^{3} \\
+2.0(2) \times 10^{-6} \quad J(J+1) \\
+7.3(8) \times 10^{-8}\left(v^{\prime}+1 / 2\right)^{2} \quad J(J+1)\end{array}$ & $\begin{array}{l}0.091 \text { (fixed) } \\
+9 .(4) \times 10^{-4} \\
\left(v^{\prime \prime}+1 / 2\right)\end{array}$ \\
\hline
\end{tabular}

Figures $3 \mathrm{a}$ and $3 \mathrm{~b}$ respectively show examples of the $\Delta e q Q$ and $\Delta C_{\mathrm{I}}$ variations, measured line by line and calculated from the above parameters. In particular, figure $3 a$ shows that this polynomial description of $\Delta e q Q$ can be safely extrapolated to the $v^{\prime}=8,9$, and even 10 results, except for a very localized perturbation around $\left(v^{\prime}=9, J=7\right)$. This perturbation in the $e q Q(\mathrm{~B})$ values takes place precisely at the first predissociation threshold in $v_{\mathrm{B}}=9$, which is now under study in our group and appears to be due to a $\Omega=0^{+}-0^{-}$ hyperfine interaction [15]. On the contrary, in figure $3 \mathrm{~b}$ it is seen that the $\Delta C_{1}$ values are :

- very insensitive to the $v_{\mathrm{X}}$ quantum number, which is also shown by the very small influence of the best-fitted $\left(v_{\mathrm{X}}+1 / 2\right)$ contribution to $C_{\mathrm{X}}$, 

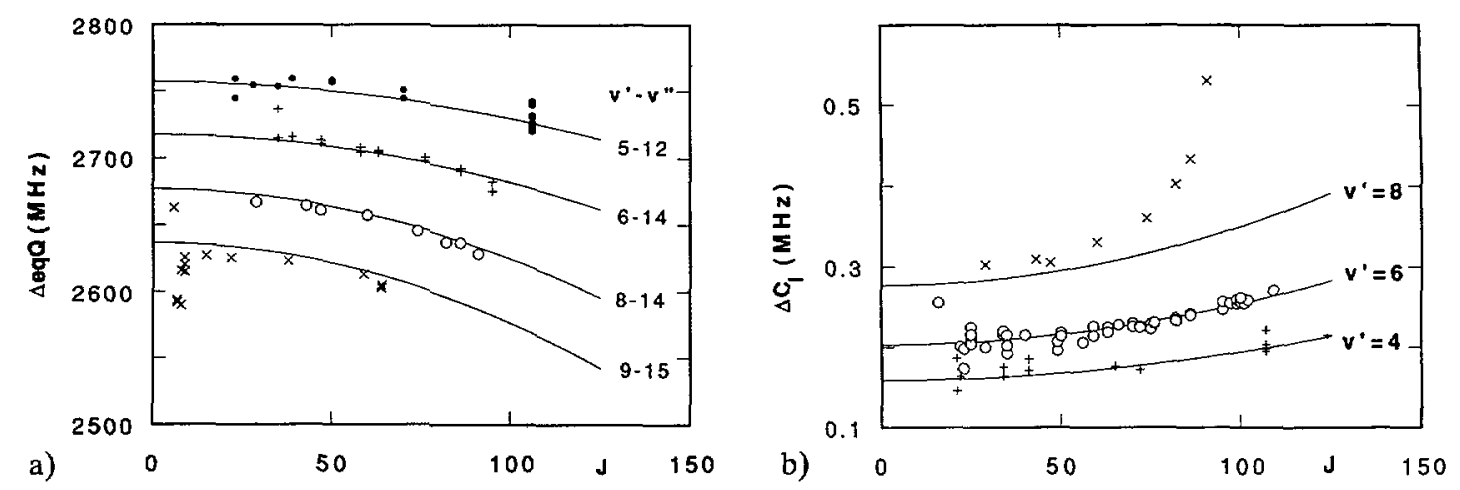

Fig. 3. - a) Plots of $\Delta e q Q$ values : the continuous lines are calculated using table II parameters. Results from a line by line fitting : $(x): 9-15$ band, $(0): 8-14$ band, $(+): 6-14$ band, $(\bullet): 5-12$ band. The $v^{\prime}=9$ results are displayed to show the influence of perturbation. b) Plots of $\Delta C_{1}$ values : the continuous lines are calculated using table II parameters. Results from a line by line fitting of the parameters : $(+): v^{\prime}=4, v^{\prime \prime}=10,11,12,15 ;(0): v^{\prime}=6, v^{\prime \prime}=13,14,17 ;(\times): v^{\prime}=8$, $v^{\prime \prime}=14$. For $v^{\prime}=4$ and 6 we have gathered results from several bands, as the $v^{\prime \prime}$ dependence of $\Delta C_{\mathrm{I}}$ is not apparent on the graph. The results of the $v^{\prime}=8$ band have been plotted, despite the fact they are not included in the fit, to demonstrate the perturbation in this state.
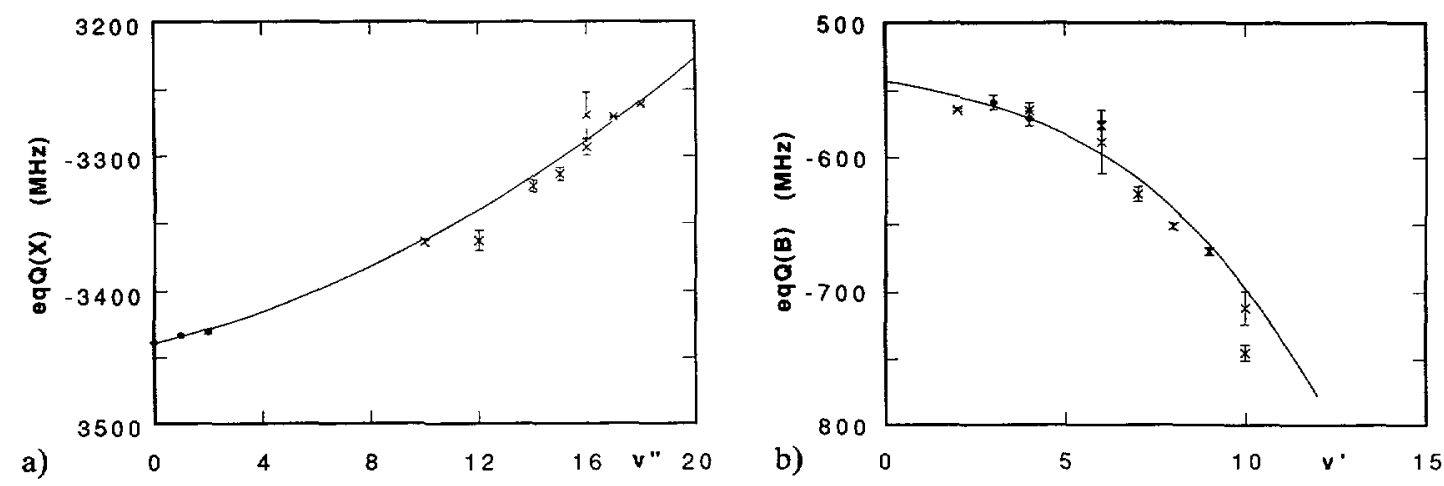

Fig. 4. - a) Vibrational variations of $e q Q$ in the IF X state; $(x)$ : our results, $(\bullet)$ : data from reference [3]. The continuous curve is obtained with the constants from table II. b) Vibrational variations of $e q Q$ in the IF B state; points : $(x)$ : our results, $(\bullet):$ data from reference [4]. The continuous curve is obtained with the constants from table II.

- increasing dramatically with $J$ for $v^{\prime}=8$, which indicates also a perturbation (in this case by a $\Omega=1$ state); this is the reason of our failure to describe the ensemble of our results by polynomials.

The fact that the $C_{\mathrm{I}}$ values are very sensitive to perturbations has already been noticed, for example in the $\mathrm{IBr} \mathrm{B}^{\prime} 0^{+}$predissociated state [16].

Our results corresponding to the $J=1$ levels are shown in figures $4 \mathrm{a}$ and $4 \mathrm{~b}$. Here are plotted the purely vibrational variations of the electric quadrupole constants $e q Q$, respectively in the $\mathrm{X}$ and $\mathrm{B}$ states, measured and also calculated from the table II parameters. 


\section{Discussion.}

We are now going to discuss the values of the IF hyperfine parameters and their vibrational and rotational dependence.

5.1 THE ElECTRIC QUADRUPOLE CONSTANT eqQ. - The electric quadrupole coupling constant is known to be very sensitive to the ionic character of the molecular bond [5]. The case of the halogen atoms, with a $\mathrm{p}^{5}$ shell is particularly nice in this respect, as the effect appears to be symmetrical for positive and negative ions in the interhalogen molecules [6]. When a negative ion is formed, with a filled $\mathrm{p}^{6}$ outer shell, the electric field gradient at the nucleus should almost vanish (the by far dominant contribution comes from the $p$ electrons when they are close to the nucleus and the long range effect of the positive ion is negligible). When a positive ion is formed, with a $\mathrm{p}^{4}$ outer shell, the electric field gradient is due to two holes in the $\mathrm{p}$ shell. If these two holes are in atomic orbitals of the same symmetry, the electric field gradient is expected to be doubled with respect to its value in a covalent bond. The assumption concerning the symmetry of the orbitals is reasonable for the ground state $X$ of the interhalogens: their molecular orbital description, limited to the valence p electrons, is : $\sigma^{2} \pi^{4} \pi^{* 4} \sigma^{* 0}$ The ionic character of the bond appears through the mixing of the $\sigma \sigma^{*}$ and $\pi \pi^{*}$ orbitals. On the positive ions, the two holes are of the same p $\sigma$ symmetry, as expected. The evolution of the electric quadrupole coupling constant has been studied in more complex molecules like $\mathbb{I F}_{5}$, where the iodine $\mathrm{p}$ shell is very depleted : in this case $e q Q$ has even a sign opposite to the one observed in $\mathrm{I}_{2}$ [17].

From this discussion, we can relate the value of the quadrupole coupling constant $e q Q^{\prime \prime}$ in the $\mathrm{X}$ state to the ionic character of this state. Writing the wavefunction in the simplest, approximate, form [7] :

$$
|\psi\rangle=\alpha\left|\psi_{\text {cov }}\right\rangle+\beta\left|\psi_{\text {ion }}\right\rangle
$$

with $|\alpha|^{2}+|\beta|^{2}=1$, we consider only the ionic configuration of the $\mathrm{I}^{+} \mathrm{F}^{-}$type. The corresponding quadrupole coupling constant is given by :

$$
e q Q^{\prime \prime}=-2 e q Q_{\text {at }}\left(1+|\beta|^{2}\right) \times(1.05)
$$

there $e q Q_{\text {at }}$ is the value of the iodine atomic quadrupole coupling constant $\left(e q Q_{\mathrm{at}}=1146,356(10) \mathrm{MHz}\right)$ [18], and the 1.05 factor is empirical, deduced from figure 3 of reference [6]. This factor expresses the enhancement of the electric field gradient due to bonding. We expect $e q Q^{\prime \prime}$ to vary rapidly with the internuclear distance, as the $|\beta|^{2}$ parameter should decrease to zero at large $r$.

It is well known that the variation $e q Q(r)$ can be used to deduce $e q Q(v, J)$ :

$$
e q Q(v, J)=\langle\mathrm{X}, v, J|e q Q(r)| \mathrm{X}, v, J\rangle
$$

where $|\mathrm{X}, v, J\rangle$ designates the radial wavefunction of the rovibrational $(v, J)$ level in the $\mathrm{X}$ state. Conversely, from a good knowledge of $e q Q^{\prime \prime}(v, J)$ one can in principle deduce the $e q Q^{\prime \prime}(r)$ variation by a RKR-type inversion due to Watson [19]. Unfortunately, our primary results give the vibrational dependence of $e q Q^{\prime \prime}$, and we have only the rotational dependence of $\Delta e q Q=e q Q^{\prime}-e q Q^{\prime \prime}$, and not the dependence of each of the parameters. Nevertheless, the vibrational dependence alone is able to give a first approximation of $e q Q^{\prime \prime}(r)$ : using only the measurements resulting from the analysis of the $P(1)$ lines, we have fitted the experimental eqQ" $(v, J=0)$ by expressing $e q Q^{\prime \prime}(r)$ as a polynomial in $\xi=\left(r-r_{\mathrm{e}}\right) / r_{\mathrm{e}}$ :

$$
e q Q^{\prime \prime}(r)=\sum_{i=0}^{2} a_{i} \xi^{i}
$$


In facts, we have been able to obtain a rather well defined set of $a_{\imath}$ values, by using the precise experimental data of reference [3], together with ours. These low $J$ data are fitted with a r.m.s. deviation of $2.7 \mathrm{MHz}$ by the following expression:

$$
e q Q^{\prime \prime}(r)=-3440.9(5)-402 .(247) \xi+7700 .(850) \xi^{2}
$$

The large uncertainty in the $\xi$ coefficient comes from the absence of high $J$ data; nevertheless, in the $r$ range investigated, this does not induce too large deviations in $e q Q^{\prime \prime}(r)$. From this fit, using equation (8), we can calculate the ionic character $|\beta|^{2}$ of the IF bonds as a function of the internuclear distance.

It is possible to compare this result with a more classical measurement of the ionic character of a molecular bond, which is the dipole moment. The dipole moment of IF in the X state has been measured $(\mu=1.948(20) \mathrm{D})$ [20], and also calculated : $\mu\left(r=r_{\mathrm{e}}\right)=1.87 \pm 0.2 \mathrm{D}$ [21]. These two results are in good agreement; moreover the calculation [21] gives the dipole moment as a function of the internuclear distance $r$. These data appear in figure 1 of reference $[21,22]$.

It is well known [23] that the polarization of the ions induce important corrections to the calculation of the actual dipole moment. Rather than using the corrections suggested by Rittner [23], we prefer to follow Nair et al. [20] :

$\mu_{\text {obs }}=\mu_{\text {Primary }}+\mu_{\text {induced }}$
where $\quad \mu_{\text {Prumary }}=|\beta|^{2} q r$

\section{$|\beta|^{2}(r)$}

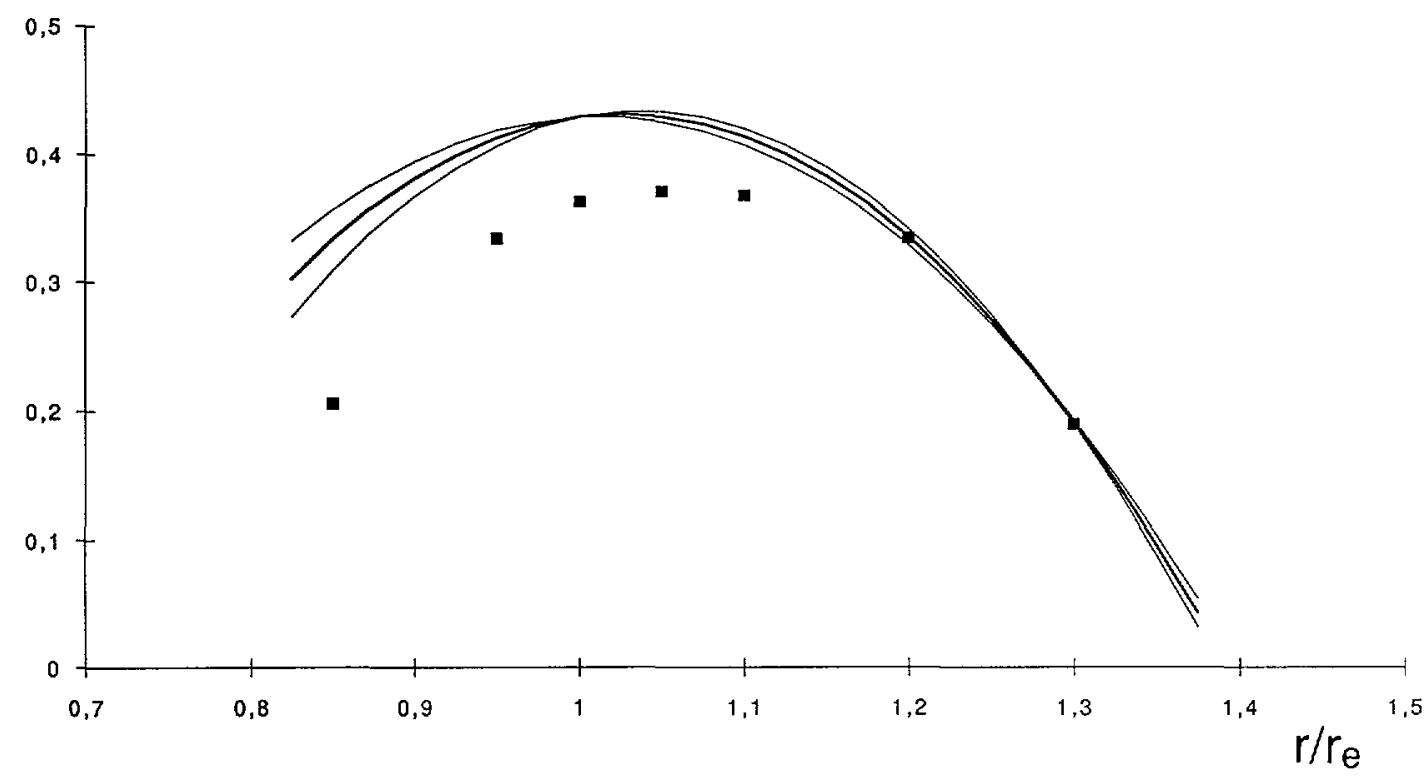

Fig. 5. - Radial dependence of the ionic mixing $|\beta|^{2}$ in the IF X state : Square dots : deduced from the calculated dipole moment values (Ref. [21]). Continuous heavy line : deduced from our eqQ" $(r)$ measurements; the light lines mark the $\pm 1 \sigma$ limits to this determination. 
and

$$
\mu_{\text {induced }}=-\mu_{\text {obs }} \times\left(\frac{\alpha_{+}}{\left[r^{2}+\left(R_{+} / 2\right)^{2}\right]^{3 / 2}}+\frac{\alpha_{-}}{\left[r^{2}+\left(R_{-} / 2\right)^{2}\right]^{3 / 2}}\right) .
$$

Here $\alpha_{+1-}$ are the polarizabilities of the ions and $R_{+1-}$ their radius. Using:

$$
\alpha\left(\mathrm{I}^{+}\right)=4 \AA^{3}[24] \text { and } \alpha\left(\mathrm{F}^{-}\right)=1.05 \AA^{3}[23] \text {, }
$$

and neglecting the $\left(R_{ \pm} / 2\right)$ terms which are small, we get a new set of $|\beta|^{2}$ values. From figure 1 of reference [21], we have copied data for $\mu_{\text {obs }}$, and calculated $\mu_{\text {Primary }}$ and thus $|\beta|^{2}$, for a number of $r / r_{\mathrm{e}}$ values. We have plotted this determination of the ionic character, together with the one deduced from equations (8) and (11), as a function of the internuclear distance $r$ in figure 5. The two sets of $|\beta|^{2}$ values agree qualitatively, showing the same maximum around $r / r_{\mathrm{e}} \approx 1$ to 1.1. The quantitative differences should not be considered too seriously, taking into account the various approximations involved in both determinations. The rapid decrease of $|\beta|^{2}$ near $r / r_{\mathrm{e}} \approx 1.3$ occurs roughly where a simple two states model (Ref. [7]) predicts. Data for longer $r$ values would be very interesting, but are not available.

A similar analysis could be tried for the variation of the B state quadrupole coupling constant. However, because the $B$ state is higher in energy, it can be mixed to various covalent and ionic states, and a simple treatment is not expected to be possible. On the other hand, from an experimental point of view, the radial $e q Q(r)$ variation is by far less well determined than in the $\mathrm{X}$ state.

5.2 The MAGNETIC HYPERFINE COUPLING CONSTANT $C_{\mathrm{I}}$ - - As already stated, the magnetic dipolar Hamiltonian $H_{\mathrm{MD}}$ has no diagonal terms inside a $\Omega=0$ state, but it couples this state to other electronic states with $\Omega=1$. These are also coupled by the Coriolis Hamiltonian $H_{\mathrm{C}}$ and the observed effect is the result of a crossed term in a second order perturbation calculation :

$$
2 \operatorname{Re} \sum_{\Omega=1 \text { states }}\left(\frac{\left\langle\Omega=0, v\left|H_{\mathrm{C}}\right| \Omega=1, v^{\prime}\right\rangle\left\langle\Omega=1, v^{\prime}\left|H_{\mathrm{MD}}\right| \Omega=0, v\right\rangle}{E_{\Omega=0}-E_{\Omega=1}}\right) .
$$

The magnitude and sign of $C_{\mathrm{I}}$ depend on the position of the $\Omega=1$ state which dominates the result. This calculation has been done in great detail in favourable cases [25], but in the present study, many $\Omega=1$ states may contribute (they are eight $\Omega=1$ states correlating to the atomic limits $\left.{ }^{2} \mathrm{P}_{J}+{ }^{2} \mathrm{P}_{J^{\prime}}\right)$. The existence of a clear perturbation in the $\mathrm{B}$ state $v=8$, is probably due to one of the states converging to the lowest atomic limits $\mathrm{I}\left({ }^{2} \mathrm{P}_{3 / 2}\right)+\mathrm{F}\left({ }^{2} \mathrm{P}_{J}\right)$, but its identification is certainly not easy.

The same calculation could also be applied to the spin-rotation term of the Fluorine nucleus : $C_{\mathrm{F}} \mathbf{I}_{2} \cdot \mathbf{J}$. The atomic magnetic hyperfine terms are comparable in fluorine and iodine [26], so that the fact that $C_{\mathrm{F}}$ is very weak deserves some explanation. The first point is that our measurements give only $\Delta C_{\mathrm{F}}=C_{\mathrm{F}^{\prime}}-C_{\mathrm{F}^{\prime \prime}}$ and do not inform us on the individual values. However, the very precise millimeter spectroscopic data [3] have not revealed any contribution from these terms, which should thus be extremely small. If IF was represented by a purely ionic configuration $\mathrm{I}^{+} \mathrm{F}^{-}$, then $\mathrm{F}^{-}$would be in a ${ }^{1} \mathrm{~S}_{0}$ state for which magnetic hyperfine interactions vanish. This is very probably part of the explanation, but the weight of the ionic configuration is at most $40 \%$ of the wavefunction; we have thus no definite interpretation of this observation. 


\section{Conclusion.}

We have measured systematically the hyperfine structure of many lines belonging to the IF B$\mathrm{X}$ system. We have used saturation spectroscopy to get rid of the Doppler effect and resolve the excitation spectra of the different hyperfine components of the rotational lines. The IF sample was produced by the $F+I_{2}$ reaction, so that we have been able to study many vibrational levels in the $X$ state, at high rotational quantum numbers. The analysis of these spectra has given the hyperfine constants $e q Q$ and $C_{\mathrm{I}}$ in the $\mathrm{B}$ and $\mathrm{X}$ states as a function of $v$ (and also the rotational dependence of the differences $\Delta e q Q$ and $\Delta C_{\mathrm{I}}$ ). The vibrational variations of some of these constants is rapid. We have discussed in detail the quadrupole coupling constant in the $X$ state, as a sensitive probe of the ionic character of the IF molecular bond.

\section{Acknowledgments.}

We thank Dr. J. P. Daudey for very helpful discussions. We gratefully acknowledge financial support from NATO and GRECO CNRS $n^{\circ} 87$ ( Dynamique des Réactions Moléculaires »). Finally, it is an honour for us to present this work within a volume dedicated to Professor Jacquinot. Since a very long period, and in great part following his impulsion, there has been deep human and scientific links between Laboratoire Aimé Cotton and ours. Retaining only the aspects we are most familiar with, we can say that : much has been done there on the halogen molecules $\left(\mathrm{I}_{2}, \mathrm{Br}_{2}\right)$, and we study here the interhalogen $\mathrm{IF}$; on another hand, atomic hyperfine structures have been the playground of Laboratoire Aimé Cotton, and we study here molecular hyperfine structures as tests of molecular bonding.

\section{References}

[1] Girard B., Billy N., Gouḱdard G. and Vigut J., Europhys. Lett. 14 (1991) 13 ; J. Chem. Phys. 95 (1991) 4056.

[2] Tiemann E., Hoeft J. and Tórring T., Z. Naturforschung 28a (1973) 1405 ;

MC Gurk. J. C. and Flygare W. H., J. Chem. Phys. 59 (1973) 5742.

[3] HoEfT J. and Natr K. P. R., Z. Phys. D 8 (1988) 85.

[4] Hartmann H. D., Knockel H. and Tiemann E., Chem. Phys. Lett. 113 (1985) 364.

[5] Townes C. H. and Schawlow A. L., Microwave Spectroscopy (McGraw-Hill New York, 1955).

[6] Tremann E., J. Mol. Struct. 97 (1983) 331.

[7] Grice R. and Herschbach D. R., Mol. Phys. 27 (1974) 159.

[8] Hansen S. G. and Howard B. J., Chem. Phys. Lett. 85 (1982) 249.

[9] Girard B., Billy N., Gouédard G. and Vigué J., J. Chem. Phys. 88 (1988) 2342.

[10] Fehsenfeld F. C., Evenson K. M. and Broida H. P., Rev. Sci. Inst. 36 (1965) 294 ; cavity n 5.

[11] Sorem M. S. and Schawlow A. L., Opt. Commun. 5 (1972) 148.

[12] Gouédard G., Billy N., Girard B. and Vigué J., Mol. Phys. 62 (1987) 1371 and 71 (1990) 913.

[13] EdMonds A. R., Angular Momentum in Quantum Mechanics (Princeton University Press, Princeton, New Jersey, 1974).

[14] Schlossberg H. R. and Javan A., Phys. Rev. 150 (1966) 267 ;

Hall J. L. and Bordé C., Phys. Rev. Lett. 30 (1973) 1101.

[15] Gouédard G., Billy N., Girard B. and Vigué J., Europhys. Lett. (submitted).

[16] Siese M. and Tiemann E., Z. Phys. D 7 (1987) 147.

[17] Shea J. C. and Kukolich S. G., Chem. Phys. Lett. 168 (1990) 489.

[18] Jaccarino V., King J. G., Satten R. A. and Stroke H. H., Phys. Rev. 94 (1954) 1798. 
[19] Watson J. K. G., J. Mol. Spectros. 74 (1979) 319.

[20] Nair K. P. R., Hoeft J. and Tiemann E., Chem. Phys. Lett. 60 (1979) 253.

[21] Kucharski S. A., Noga J. and Bartlett R. J., J. Chem. Phys. 88 (1988) 1035.

[22] Note : in figure 1 of reference [21], the horizontal scale is incorrect and readings should be diminished by 0.05 (KUCHARSKI S. A., private communication, 1989).

[23] Rittner E. S., J. Chem. Phys. 19 (1951) 1030.

[24] BRand J. C. D. and Hoy A. R., J. Mol. Spectros. 97 (1983) 379.

[25] Vigue J., Broyer M. and Lehmann J. C., Phys. Rev. Lett. 42 (1979) 883.

[26] ${ }^{127} \mathrm{I}: a\left({ }^{2} \mathrm{P}_{12}\right)=6591$.(6) $\mathrm{MHz}$; LUC-KENIG E., MORILlon C. and Vergès J., Physica 70 (1973) 175.

${ }^{19} \mathrm{~F}: a\left({ }^{2} \mathrm{P}_{1 / 2}\right)=10244.21(3) \mathrm{MHz}$; Harvey J. S. M., Proc. R. Soc. A 285 (1965) 581. 